20

\title{
Апробация тонкоигольного оптического зонда для регистрации изменений флуоресценции коферментов клеточного дыхания
}

\author{
() К.Ю. Кандурова ${ }^{1}$, Е.В. Потапова ${ }^{1}$, Е.А. Жеребцов ${ }^{1,2}$, В.В. Дрёмин ${ }^{1,2}$, Е.С. Серёгина ${ }^{1}$, \\ А.Ю. Винокуров ${ }^{3}$, А.В. Мамошин ${ }^{1,4}$, А.В. Борсуков ${ }^{5}$, Ю.В. Иванов ${ }^{6,7}$, А.В. Дунаев ${ }^{1}$
}

${ }^{1}$ Научно-технологический центр биомедицинской фотоники,

Орловский государственный университет имени И.С. Тургенева,

302026 Орел, Россия

${ }^{2}$ University of Oulu,

90570 Oulu, Finland

${ }^{3}$ Кафредра промышленной химии и биотехнологии, Орловский государственный университет имени И.С. Тургенева, 302026 Орел, Россия

${ }^{4}$ Орловская областная клиническая больница,

302028 Орел, Россия

${ }^{5}$ Проблемная научно-исследовательская лаборатория „Диагностические исследования и малоинвазивные технологии“, Смоленский государственный медицинский университет,

214006 Смоленск, Россия

${ }^{6}$ Федеральный научно-клинический центр специализированных видов

медицинской помощи и медицинских технологий ФМБА России,

115682 Москва, Россия

${ }^{7}$ Центральный научно-исследовательский институт туберкулеза,

107564 Москва, Россия

e-mail: kandkseniya@gmail.com

Поступила в редакцию 10.12.2019 г.

В окончательной редакции 31.01.2020 г.

Принята к публикации 28.02.2020 г.

Описано устройство для оптической биопсии с каналом флуоресцентной спектроскопии и тонкоигольным оптическим зондом для применения при тонкоигольной пункционно-аспирационной биопсии новообразований печени. Для апробации разработанного устройства проведены экспериментальные измерения флуоресценции внутренних органов лабораторной крысы in vivo при воздействии на поверхности тканей митохондриального разобщителя для индуцирования изменений клеточного дыхания. Полученные результаты постановочного эксперимента показали способность разработанного канала регистрировать изменения флуоресценции, обусловленные изменениями в процессах окислительного фосфорилирования митохондрий.

Ключевые слова: оптическая биопсия, флуоресцентная спектроскопия, митохондриальные разобщители, НАДН, ФАД.

DOI: $10.21883 /$ OS.2020.06.49405.32-20

\section{Введение}

Согласно статистике Всемирной организации здравоохранения, онкологические заболевания являются второй ведущей причиной смерти в мире [1]. Одним из труднодиагностируемых видов онкопатологии является рак печени. Первичный рак печени является шестым по распространенности в мире и четвертым по летальности среди остальных видов злокачественных новообразований [2,3]. При этом наблюдается тенденция к увеличению числа зарегистрированных случаев данного заболевания $[4,5]$. Также известно, что печень является органом, в котором чаще всего возникают метастазы, обусловленные злокачественными новообразованиями в других органах [6]. Одним из факторов, улучшающих прогноз у пациентов с опухолями печени, является возможность более ранней диагностики.
Несмотря на современный технический и методологический уровень медицины, диагностика рака печени до сих пор вызывает определенные трудности. „Золотым стандартом“ предоперационной диагностики злокачественных опухолей является гистологическое и цитологическое исследования образцов тканей и клеток [7]. Для их получения проводится процедура тонкоигольной пункционно-аспирационной биопсии (ТПАБ) $[8,9]$, при которой образец ткани извлекается из нескольких зон исследуемого патологического очага с помощью тонкой иглы с обычным или режущим краем под контролем таких методов визуализации, как ультразвуковое исследование, компьютерная томография или магнитнорезонансная томография. Среди преимуществ данной процедуры отмечаются миниинвазивность, атравматичность, низкая вероятность возникновения осложнений, высокая точность и экономическая эффективность [10]. 
Материал, полученный во время ТПАБ, отправляется на плановое гистопатологическое и цитологическое исследования, результаты которых врач-хирург получает только через 5-10 дней после процедуры, в то время как получение информации о состоянии тканей пораженного органа в этот период представляет интерес с точки зрения выбора тактики дальнейшего лечения. Другой проблемой метода является вероятность забора неинформативного материала из-за ряда причин: физиологическое смещение органов, манипуляции, предшествующие ТПАБ, непроизвольные движения пациента, недостаточная визуализация очага новообразования изза его небольших размеров и гетерогенности. Указанные выше факторы приводят к тому, что даже опытные хирурги допускают до 15-29\% недиагностических образцов [11-13]. Ложноотрицательный результат приводит к необходимости повторной процедуры, что может быть связано с риском развития осложнений для пациента и увеличивает длительность лечения. Таким образом, для повышения эффективности лечения пациентов со злокачественными новообразованиями печени актуальной задачей является разработка и внедрение новых методов диагностики, позволяющих получать информацию в режиме реального времени.

Одной из быстро развивающихся областей приложения биомедицинской оптики для решения задач хирургии являются методы оптической биопсии [14], которые объединяют в себе преимушества традиционной биопсии и компенсируют ее главный недостаток - необходимость изъятия образца ткани и значительные затраты времени на анализ. Оптическая биопсия включает в себя ряд методов спектроскопии и визуализации $[15,16]$, позволяющих получать дополнительную диагностическую информацию о различных параметрах морфологии и метаболизма биологических тканей в режиме реального времени in vivo $[17,18]$. В частности, для проведения оптической биопсии во время ТПАБ с использованием игл малого наружного диаметра (14-22 G, или 0.7-2.1 mm) самым распространенным направлением доклинических исследований является разработка тонкоигольных оптических зондов для реализации различных спектроскопических методов диагностики [19-24].

Среди оптических методов диагностики широкое применение для исследования метаболической активности клеток здоровых и патологически измененных тканей (воспаленная, злокачественная ит.д.) в ряде областей медицины нашла флуоресцентная спектроскопия (ФС) $[25,26]$. Данный метод основан на возбуждении флуоресценции эндогенных или экзогенных флуорофоров в биологической ткани монохроматическим излучением ближнего ультрафиолетового (УФ) или видимого диапазонов и дальнейшей регистрации полученного спектра для анализа и сравнения. В ряде работ флуоресцентная спектроскопия используется как основной диагностический метод или в составе мультимодальных устройств, имеющих тонкоигольные оптоволокон- ные зонды для диагностики опухолевых образований легких [27,28] и молочных желез [29,30].

Многие формы коферментов никотинамидадениндинуклеотида (НАД) и флавинадениндинуклеотида (ФАД), содержащиеся в цитозоле и митохондриях клеток, обладают выраженными спектрами эндогенной флуоресценции, изменения в интенсивности которых возможно регистрировать in vivo, что лежит в основе исследований метаболической активности тканей [31-34]. Указанные коферменты являются неотъемлемой частью реакций клеточного метаболизма, выступая в качестве доноров и акцепторов электронов для синтеза молекул аденозинтрифосфата (АТФ), обеспечивающих энергией прочие многочисленные биохимические реакции [35]. Оба кофермента претерпевают реакции окисления и восстановления, при этом из всех форм наибольший вклад в формирование спектров флуоресценции тканей вносят восстановленный НАД (НАДН) и окисленный ФАД. Проведенные ранее клинические исследования показали, что изменения интенсивностей флуоресценции НАДН и ФАД в тканях связаны с возникновением патологических процессов в них, в том числе с развитием онкологических процессов [36]. Однако спектр флуоресценции тканей, регистрируемый спектрометром, является следствием сложения сигналов флуоресценции не только НАДН и ФАД, но и других эндогенных флуорофоров, таких как коллаген, эластин, порфирины, липофусцин, билирубин и др. Флуоресценция этих веществ также возбуждается светом с длинами волн, близкими к длинам волн возбуждения окислительно-восстановительных коферментов. По этой причине необходимо учитывать вклад НАДН и ФАД в общий регистрируемый сигнал флуоресценции для правильной интерпретации различий, наблюдаемых между здоровыми и патологически измененными тканями.

Разработка канала ФС для оптической биопсии злокачественных новообразований требует надежных и воспроизводимых измерений спектральных характеристик нормальных и патологических тканей в области исследования. Для дальнейшего внедрения технологии в клиническую практику необходимо проводить исследования, направленные на оценку чувствительности устройств оптической биопсии к изменениям сигнала флуоресценции, отражающим метаболическую активность в митохондриях клеток, а также на разработку методик корректировки регистрируемого спектра для более точной интерпретации данных. Распространённым способом апробации устройств флуоресцентной диагностики в настоящее время является использование специально разрабатываемых тест-объектов (фантомов). Такие фантомы обладают близкими к биологическим тканям оптическими свойствами и изготавливаются для имитации спектральных характеристик, определяемых содержанием различных флуорофоров [37-39]. Другим направлением являются in vitro и in vivo исследования оценки влияния факторов, способных вызвать изменения метаболической активности в тканях. Одним из 
таких факторов является применение широкого спектра митохондриальных ингибиторов и разобщителей, оказывающих воздействие непосредственно на функции митохондрий, что отражается в аномальном увеличении или уменьшении накопления коферментов НАДН и ФАД в ходе биохимических реакций, а следовательно, дает отклик при измерении интенсивности флуоресценции [40-42]. Однако большинство протоколов подобных исследований разработаны и применяются для измерений на клеточных культурах [43-47], единичные работы посвящены исследованиям, адаптирующим данный подход к целым органам модельных животных $[32,48]$.

Таким образом, целью данной работы явилось исследование чувствительности измерительного канала ФС устройства для тонкоигольной оптической биопсии к содержанию основных целевых эндогенных флуорофоров, связанных с клеточным метаболизмом, для того чтобы объективно продемонстрировать способность разработанного устройства оценивать состояние биологических тканей in situ и in vivo.

\section{Материалы и методы}

В ходе постановочного эксперимента исследования проводились на специально разработанном устройстве для проведения оптической биопсии [49], содержащем два измерительных канала - ФС и спектроскопии диффузного отражения (СДО). В канале ФС для возбуждения автофлуоресценции НАДН и ФАД использовалось излучение от светодиода с длиной волны $365 \mathrm{~nm}$ и лазерного диода с длиной волны $450 \mathrm{~nm}$ [50]. Выходная мощность используемых источников составила не более $1.5 \mathrm{~mW}$ и $3.5 \mathrm{~mW}$ соответственно, что обеспечивает соблюдение требований безопасности по эффективной освещенности тканей [51,52], а также уменышает эффект фотообесцвечивания коферментов. Для ослабления обратно рассеянного излучения источников использовались светофильтры FGL400 и FGL495 (Thorlabs, Inc., USA) с длинами волн среза $400 \mathrm{~nm}$ и $495 \mathrm{~nm}$ в соответствии с используемыми источниками. Для регистрации спектров флуоресценции в диапазоне 350-1000 nm использовался малогабаритный ПЗС-спектрометр FLAMET-VIS-NIR-ES (Ocean Optics, USA).

Канал СДО необходим для компенсации влияния кровенаполнения тканей на сигнал флуоресценции, а также может быть использован для получения дополнительной информации о морфологической структуре тканей. Канал содержит широкополосный вольфрамовый галогенный источник излучения HL-2000-FHSA (Ocean Optics, USA) с диапазоном 360-2400 nm. Управление устройством и обработка получаемых данных осуществлялись с помощью специально разработанного программного обеспечения в программной среде MATLAB.

Доставка излучения от источников и сбор вторичного оптического излучения от биологической ткани осуществляется с помощью специально разработанного

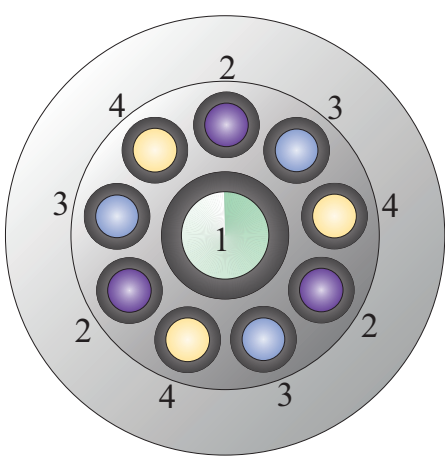

$a$

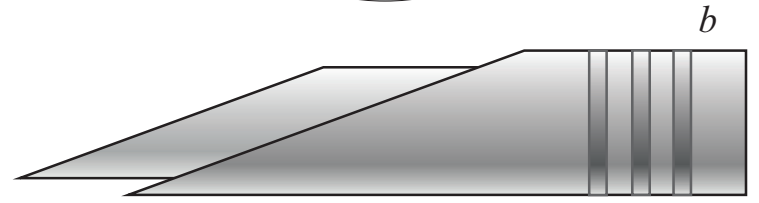

Рис. 1. (a) Схема расположения оптических волокон в тонкоигольном оптическом зонде устройства для проведения оптической биопсии: 1 - волокно к спектрометру, 2 от источника $365 \mathrm{~nm}, 3$ - от источника $450 \mathrm{~nm}, 4$ - от широкополосного источника. $(b)$ Расположение оптического зонда в стандартной игле $17.5 \mathrm{G}$.

тонкоигольного оптического зонда с наружным диаметром $1 \mathrm{~mm}$, что позволяет вводить его в исследуемую область через тонкую иглу с наружным диаметром $17.5 \mathrm{G}$ (рис. 1). Внутри зонда расположены 10 оптических волокон: центральное волокно диаметром $200 \mu \mathrm{m}$ используется для сбора излучения и его передачи к спектрометру, 9 волокон диаметром $100 \mu \mathrm{m}$ (по 3 волокна для каждого источника) служат для равномерного освещения области исследования источниками излучения во время измерений. Торец волоконного зонда имеет скос $20^{\circ}$ для обеспечения плотного контакта с тканями. Значение числовой апертуры оптических волокон в зонде - 0.22 .

Для того чтобы смоделировать быстрые изменения метаболической активности тканей, использовался разобщитель окислительного фосфорилирования (протонофор) карбонилцианид м-хлорфенил-гидразон (carbonyl cyanide $m$-chlorophenyl hydrazone, CCCP). Соединение СССР является ингибитором окислительного фосфорилирования, повышающим проницаемость митохондриальной мембраны, нарушая тем самым протонный градиент [53]. Нанесение СССР на поверхность ткани вызывает снижение содержания НАДН в клетках, содержание ФАД наоборот повышается. Нарушение переноса протонов по электронной транспортной цепи приводит к недостаточному синтезу молекул АТФ, что в целом ведёт к постепенному разрушению клеток и гибели организма [54].

Для получения исходного раствора СССР из концентрата (Sigma-Aldrich, USA) в качестве растворителя был выбран диметилсульфоксид (dimethyl sulfoxide, DMSO) [46]. Это вещество широко распространено в химических исследованиях как сероорганический рас- 


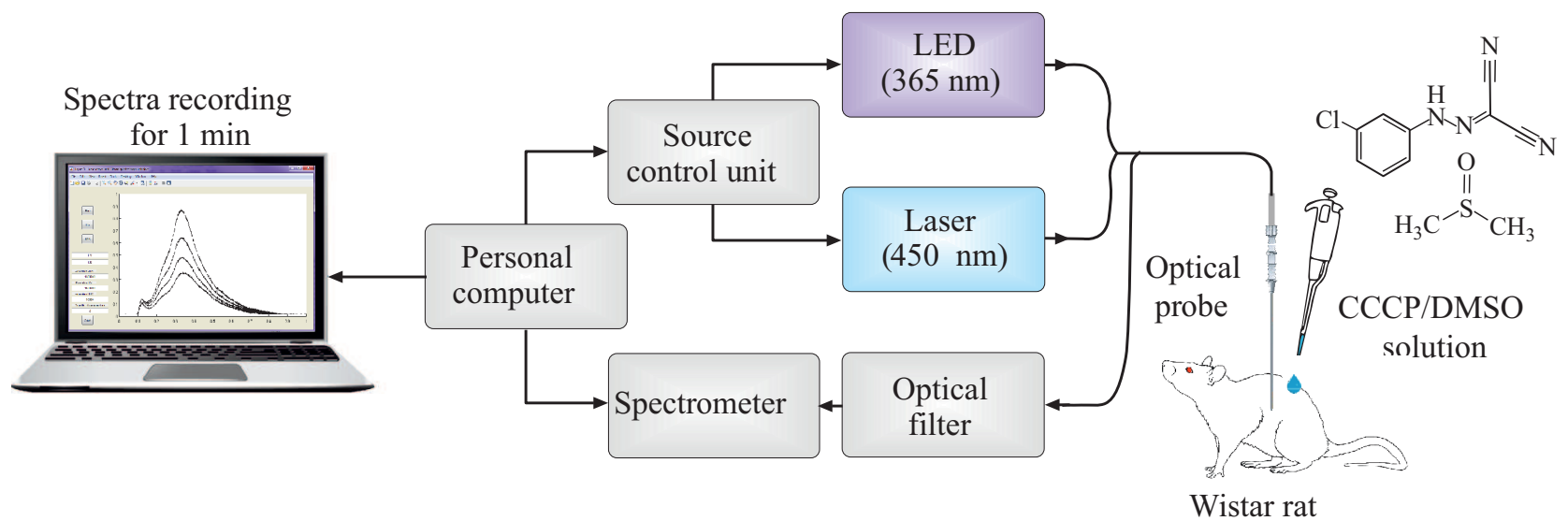

Рис. 2. Схема проведения эксперимента с помощью канала флуоресцентной спектроскопии устройства для проведения тонкоигольной оптической биопсии.

творитель для других веществ. В биофотонике DMSO применяется для оптического просветления биотканей [55], при этом просветляющие свойства DMSO сохраняет спустя длительный промежуток времени после воздействия [56]. Другой особенностью DMSO, важной для данной работы, является способность не только проникать в биологические ткани, но и переносить в них другие химические соединения [57,58], что может быть использовано для более выраженного наблюдения эффекта от использования раствора СССР.

Для подбора оптимальных значений достоверного сигнала флуоресценции ткани в зависимости от типа самой ткани и характеристик разработанного канала ФС в ходе эксперимента использовались различные концентрации раствора СССР. Для экспериментальных исследований был использован исходный раствор СССР в концентрации $1 \mathrm{mM}$ и разбавленные растворы в концентрации $0.1 \mathrm{mM}$ и $0.01 \mathrm{mM}$, полученные путем разведения в натрий-фосфатном буфере (phosphate buffered saline, PBS). Раствор DMSO использовался для проведения контрольных измерений без разобщителя в исходной концентрации $100 \%$ и в концентрациях $10 \%$ и $1 \%$ после разведения в $\mathrm{PBS}$.

В качестве модельного животного в исследовании использовался клинически здоровый самец крысы линии Wistar (возраст 3 месяца). Крысу анестезировали препаратом Золетил 100 (Vibrac, Франция) в стандартной дозе и фиксировали на специальной платформе. На первом этапе эксперимента измерения проводились после нанесения растворов DMSO и CCCP микропипеткой на предварительно подготовленной коже живота. На втором этапе выполнялась лапаротомия с последующим нанесением растворов на поверхности печени, сердца и скелетных мышц задней конечности крысы. Регистрация спектров флуоресценции проводилась с интервалом в $1 \mathrm{~s}$. Схематическое изображение эксперимента приведено на рис. 2.

После регистрации спектров флуоресценции для анализа динамики изменения сигнала во времени выби- рались максимальные значения интенсивности флуоресценции в диапазонах 480-530 nm (для источника излучения $365 \mathrm{~nm}$ ) и $500-550 \mathrm{~nm}$ (для источника излучения $450 \mathrm{~nm}$ ). Относительное изменение интенсивности флуоресценции в течение эксперимента оценивалось путем вычисления отношения интенсивности флуоресценции в $N$-ю секунду эксперимента $I F_{N}$ к значению интенсивности флуоресценции, зарегистрированному в начале измерений $I F_{0}$. По полученным соотношениям были построены кривые изменения максимальных интенсивностей флуоресценции во времени.

\section{Результаты исследований и их обсуждение}

Подбор мощностей источников излучения осуществлялся исходя из мер по снижению влияния фотообесцвечивания. В начале каждого измерения перед нанесением растворов DMSO и CCCP проводилась регистрация спектров флуоресценции при непрерывном освещении ткани для учета наличия данного эффекта. В ряде случаев наблюдалось незначительное фотообесцвечивание тканей, причем в среднем этот эффект был более выражен для источника излучения $450 \mathrm{~nm}$. После данной проверки в случае, если фотообесцвечивание не было выражено, наносились растворы веществ и проводилась регистрация массива спектров. В ходе эксперимента часть сигналов при различных концентрациях веществ, воздействующих на ткани органов, были признаны неудовлетворительными из-за низкого соотношения сигнал-шум и не были учтены при анализе данных.

При анализе динамики изменения максимальной интенсивности флуоресценции в ходе эксперимента с воздействием растворами DMSO (рис. 3,4) было отмечено, что спектры флуоресценции кожи и мышечной ткани показывают удовлетворительную воспроизводимость. При этом флуоресценция мышечной ткани оставалась 

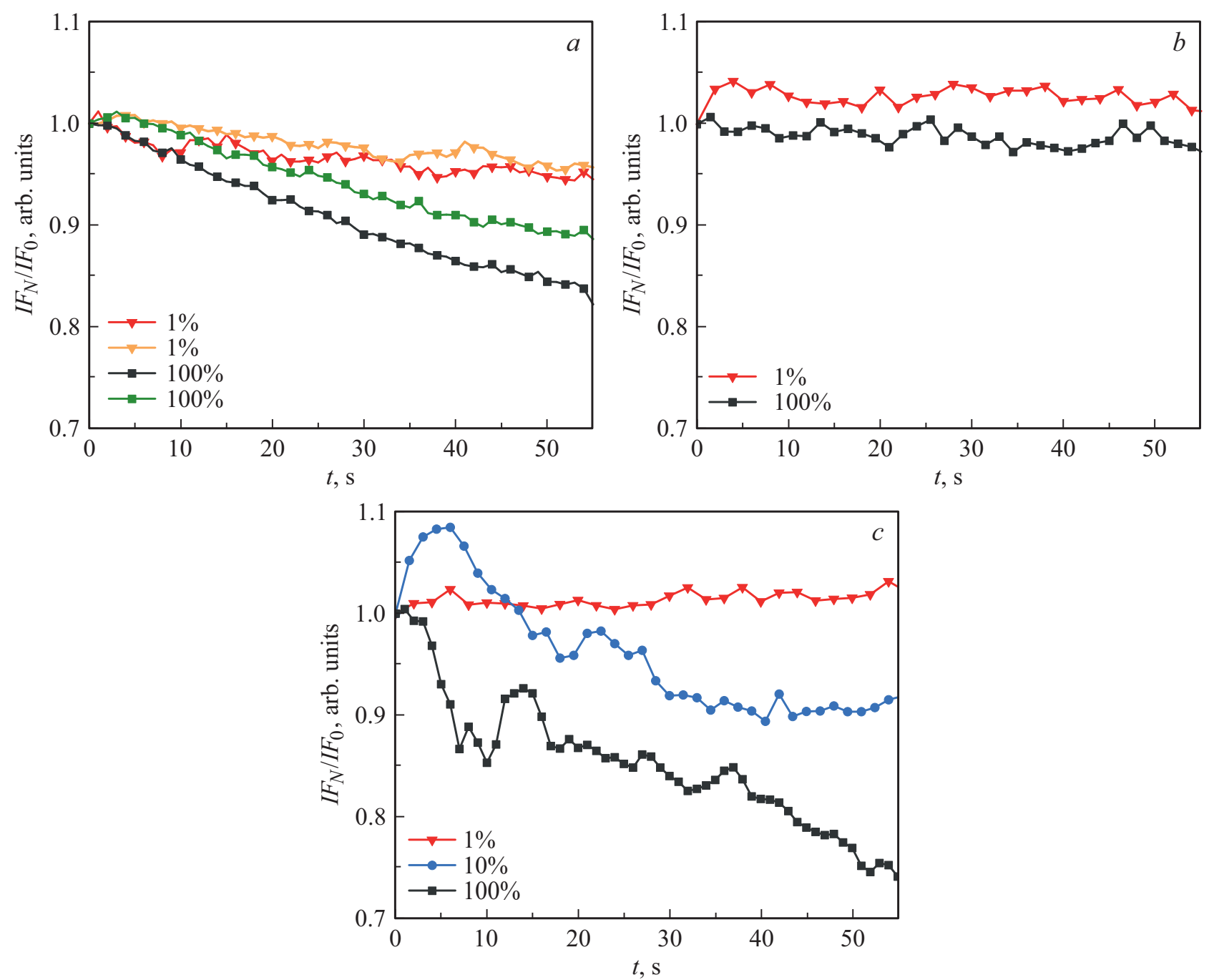

Рис. 3. Динамика изменения максимальной интенсивности флуоресценции в диапазонах $480-530 \mathrm{~nm}$ для источника излучения $365 \mathrm{~nm}$ после применения различных концентраций DMSO. Области исследования: $a-$ кожа, $b-$ мышца, $c-$ печень.

относительно стабильной (падение сигнала не более $10 \%$ ) под воздействием раствора DMSO с разными концентрациями при возбуждении излучением как $365 \mathrm{~nm}$, так и $450 \mathrm{~nm}$. Падение сигнала на коже составило $10 \%$ и более в случае взаимодействия ткани с более высокой $(100 \%)$ концентрацией DMSO. В случае внутренних органов было отмечено, что интенсивность флуоресценции в тканях печени и сердца снижалась более значительно, в частности, в печени снижение достигло более $25 \%$ (при возбуждении излучением $450 \mathrm{~nm}$ ) даже при малых концентрациях действующего агента. При возбуждении излучением $365 \mathrm{~nm}$ падение сигнала было значительным только при воздействии на ткани печени чистым раствором DMSO. Поскольку в эксперименте были предприняты меры для записи данных без влияния фотообесцвечивания, предполагается, что это может быть вызвано токсическим действием DMSO, которое может проявляться изменениями в структуре клеточной мембраны при определенных концентрациях. Так, тради- ционно считается, что в исследованиях in vivo DMSO не оказывает токсического действия при концентрациях вплоть до 10\% [59,60], но при более высоких концентрациях данное вещество проявляет свойства ингибитора митохондриального дыхания и приводит к апоптозу клеток [61,62]. Кроме того, в литературе встречаются сведения о механизмах возникновения токсического действия DMSO при использовании концентраций около $2-4 \%$ [63].

Как и при измерениях с растворами DMSO, было замечено, что ткани кожи и мышц независимо от концентрации вещества в меньшей степени проявили ожидаемый от СССР эффект в отличие от тканей печени и сердца. Возможно, это связано с тем, что СССР и DMSO имели разнонаправленное действие на флуоресцентный сигнал и частично компенсировали друг друга.

После воздействия на ткани печени раствором СССР с концентрацией $0.1 \mathrm{mM}$ было замечено, что флуоресценция, индуцированная излучением $365 \mathrm{~nm}$, уменьши- 

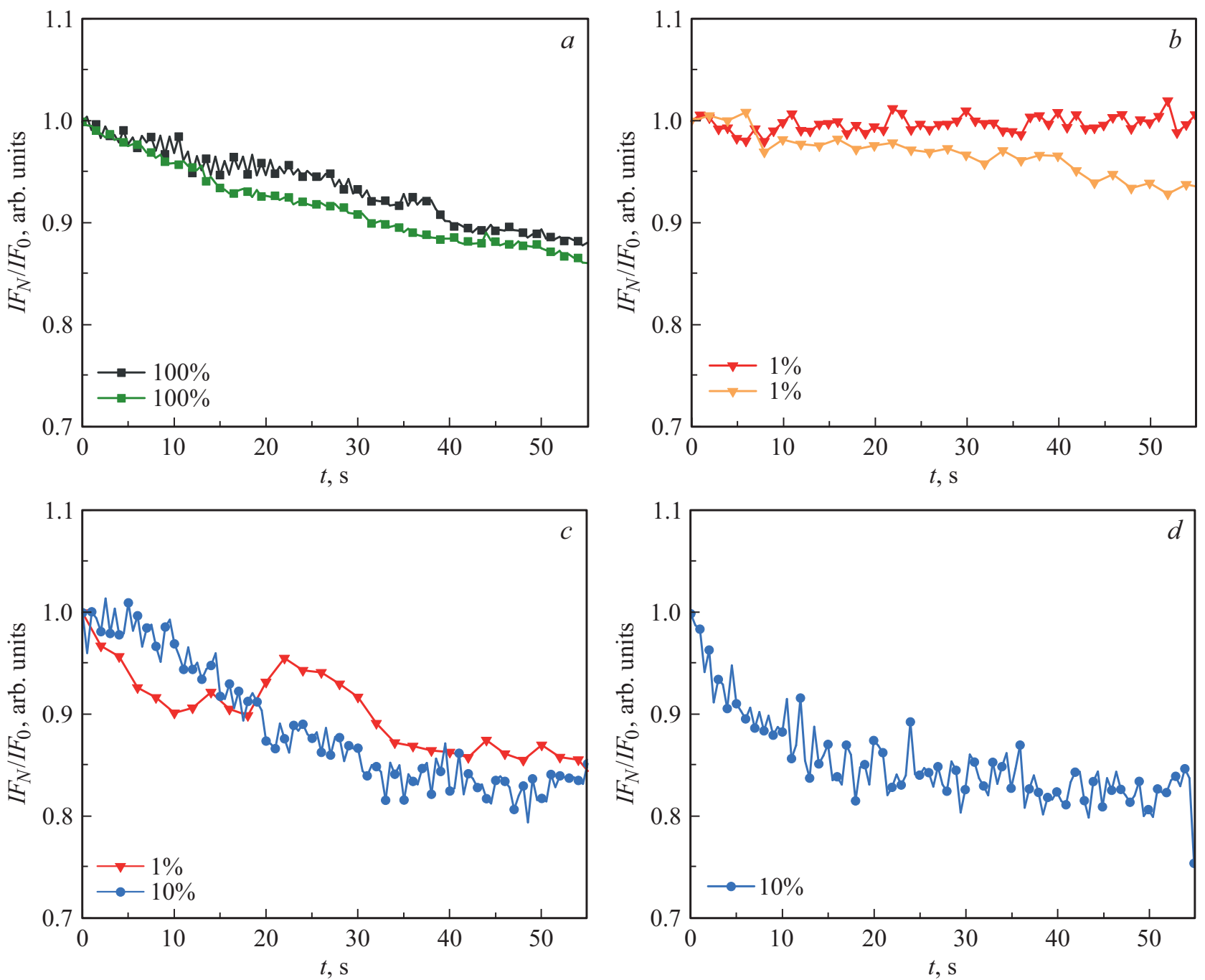

Рис. 4. Динамика изменения максимальной интенсивности флуоресценции в диапазонах $500-550 \mathrm{~nm}$ для источника излучения $450 \mathrm{~nm}$ после применения различных концентраций DMSO. Области исследования: $a-$ кожа, $b-$ мышца, $c-$ печень, $d-$ сердце.

лась так же сильно, как при воздействии $100 \%$ раствором DMSO (рис. 5,c), при этом основной процесс снижения интенсивности флуоресценции пришелся на первые 20 s. Поскольку нарушение окислительного фосфорилирования из-за разобщения протонного перехода приводит к повышенному потреблению электронов, реакции окисления донора электронов НАДН до НАД происходят более активно. Так как НАД не обладает таким же выраженным спектром флуоресценции, как НАДН, снижение содержания восстановленной формы кофермента в клетках становится заметным по характерному снижению максимальной интенсивности флуоресценции во времени [46].

При возбуждении излучением с длиной волны $450 \mathrm{~nm}$ в тканях сердца и печени нанесение раствора СССР концентрацией $1 \mathrm{mM}$ вызывало значительное увеличение флуоресценции (рис. 6). Динамика роста сигнала во времени может быть обусловлена тем, что по мере того, как СССР вызывает повышенную активность митохондрий из-за стимулирования реакций окислительного фосфорилирования и возрастает потребление электронов, все большее количество молекул восстановленной формы ФАДН2 окисляются до ФАД, что приводит к увеличению интенсивности флуоресценции [64].

Результаты экспериментальных исследований показали, что кожа и скелетные мышцы задней лапы крысы были менее восприимчивы к описанным химическим воздействиям, в то время как внутренние органы (печень, сердце) оказались более чувствительными и подверглись как ожидаемому эффекту разобщения окислительного фосфорилирования при воздействии СССР, так и токсическому эффекту растворителя DMSO. Использование разных концентраций растворов не вызвало различий в скорости и величине изменений интенсивности флуоресценции кожи и мышц, в то время как применение высоких концентраций растворов на тканях печени и 

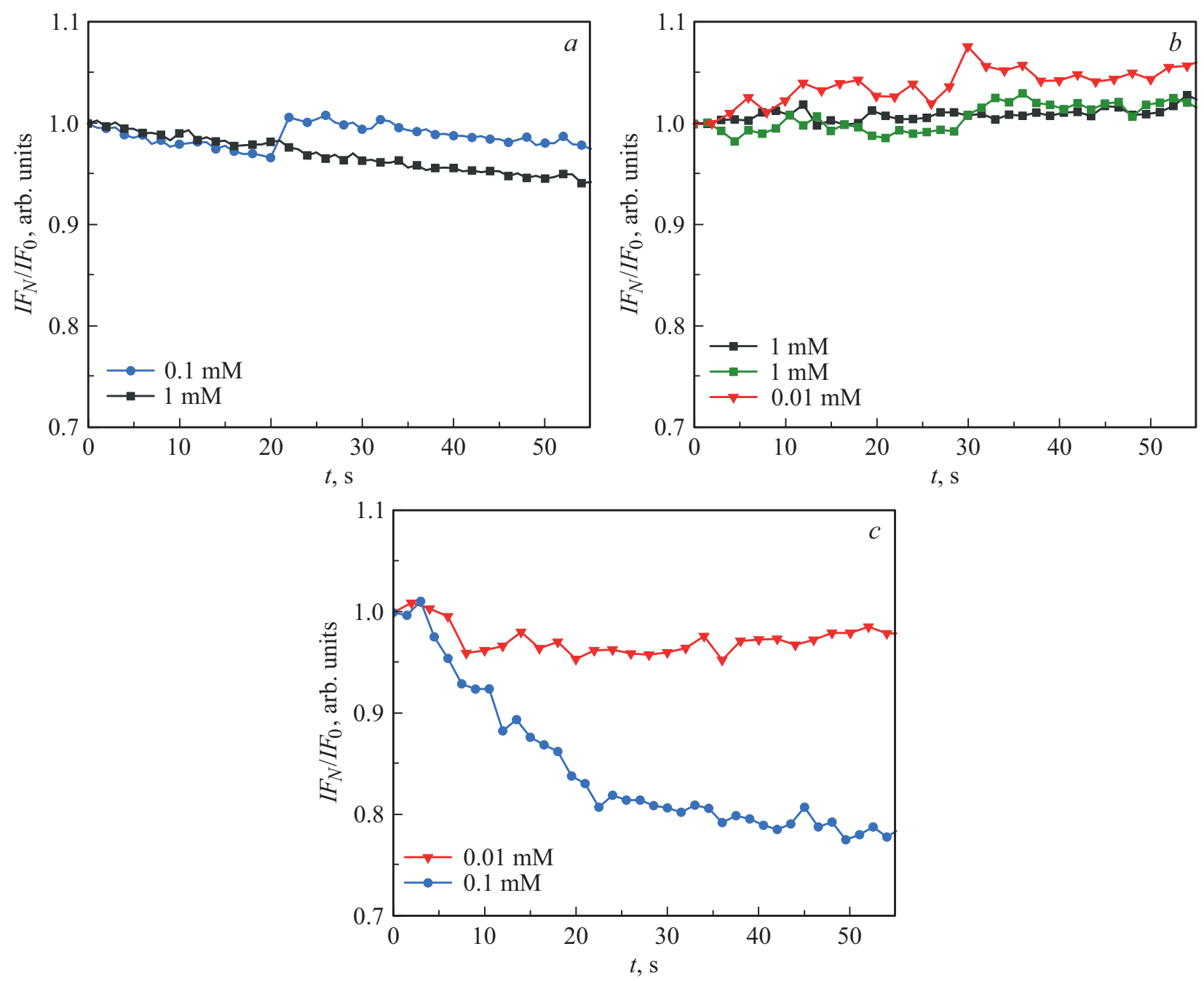

Рис. 5. Динамика изменения максимальной интенсивности флуоресценции в диапазонах $480-530 \mathrm{~nm}$ для источника излучения $365 \mathrm{~nm}$ после применения различных концентраций СССР. Области исследования: $a-$ кожа, $b-$ мышца, $c-$ печень.

сердца позволило добиться более выраженного и быстрого проявления изменений интенсивности флуоресценции, обусловленных влиянием на метаболическую активность клеток в тканях in vivo.

\section{Выводы}

Использование широкого числа методов оптической биопсии в настоящее время представляется многообещающим направлением для внедрения в клиническую практику врача-хирурга, так как они позволяют получать дополнительную диагностическую информацию о состоянии метаболизма и морфологической структуре биологических тканей в режиме реального времени, которая может иметь важное значение в процессе лечения онкологических заболеваний. В частности, для обеспечения первичной диагностической оценки новообразований печени уже в процессе проведения ТПАБ одним из перспективных методов представляется ФС, которая может быть внедрена в том числе и в стандартные биопсийные иглы. В настоящей работе была проверена чувствительность канала ФС устройства, разработанного ранее, для проведения оптической биопсии во время процедуры ТПАБ к метаболическим изменениям в митохондриях клеток in vivo. Это крайне важно для оценки процессов канцерогенеза в биологических тканях, в том числе печени, одним из отличительных признаков которого являются метаболические нарушения вследствие активного роста опухоли.

Разработка протоколов и проведение in vivo исследований влияния факторов, способных вызвать изменения в процессах окислительного фосфорилирования митохондрий, может лечь в основу более точной интерпретации данных ФС. Полученные в настоящей работе результаты показывают возможность адаптации методологии анализа клеточного метаболизма in vitro к проведению подобных измерений in vivo в тканях органов. Однако необходимы дальнейшие исследования, направленные на экспериментальный выбор оптимальных концентраций CCCP и DMSO с учетом полученных результатов, в том 

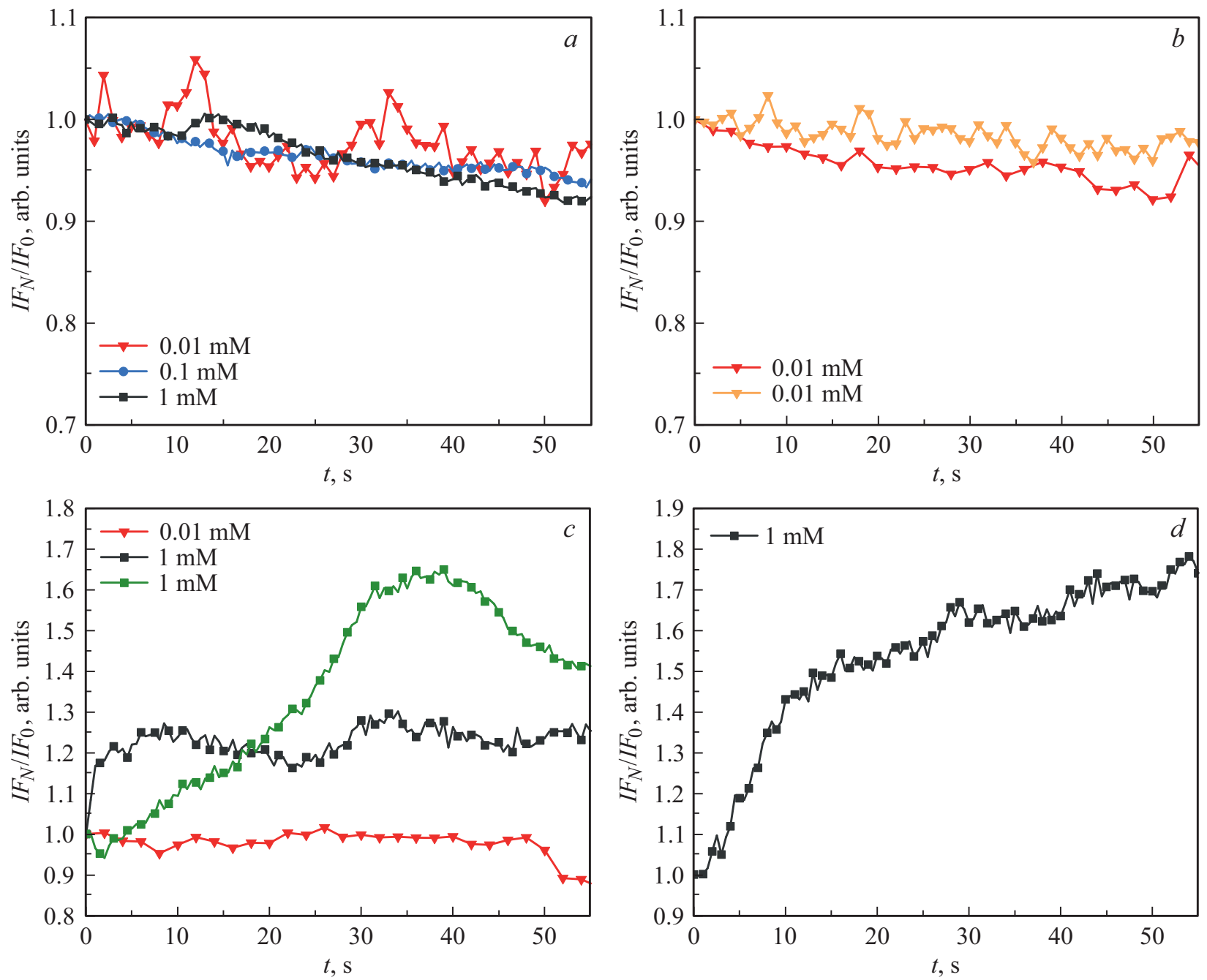

Рис. 6. Динамика изменения максимальной интенсивности флуоресценции в диапазонах $500-550 \mathrm{~nm}$ для источника излучения $450 \mathrm{~nm}$ после применения различных концентраций СССР. Области исследования: $a-$ кожа, $b-$ мышца, $c-$ печень, $d-$ сердце.

числе выводов о токсичности действия DMSO на ткани in vivo. Так как результаты, представленные в настоящей работе продемонстрировали более выраженный эффект для спектров флуоресценции, в которые наибольший вклад вносило изменение содержания ФАД, представляет интерес наблюдение подобного эффекта, вызванного изменениям НАДН. Поэтому в дальнейших исследованиях предполагается применять другие митохондриальные ингибиторы и разобщители. Для этих целей возможно использовать ингибитор ротенон, вызывающий блокирование переноса электронов через комплекс I, избыток которых отражается в быстром увеличении содержания НАДН без существенных изменений в содержании ФАД [65].

Полученные в представленной работе результаты показали способность канала ФС устройства оптической биопсии регистрировать изменения флуоресценции, обусловленные метаболическими изменениями в тканях, что подтверждает обоснованность использования устройства для проведения ТПАБ в клинической практике, в том числе при диагностике новообразований печени.

\section{Финансирование исследования}

Исследование выполнено при поддержке Российского научного фонда (проект № 18-15-00201).

\section{Соблюдение этических стандартов}

Исследования проводились в соответствии с Принципами надлежащей лабораторной практики (GLP), установленными Организацией экономического сотрудничества и развития (ОЭСР), и были одобрены этическим комитетом ФГБОУ ВО „Орловский государственный университет имени И.С. Тургенева“ (протокол заседания № 12 от 6.09.2018).

\section{Конфликт интересов}

Авторы заявляют, что у них нет конфликта интересов. 


\section{Список литературы}

[1] The Cancer Atlas, 3rd edition. International Agency for Research on Cancer. 2019. [Электронный pecypc] Режим доступа: https://canceratlas.cancer.org

[2] Ferlay J., Soerjomataram I., Ervik M., Dikshit R., Eser S., Mathers C., Rebelo M., Parkin D.M., Forman D., Bray F. // Lyon, Fr. Int. agency Res. cancer. 2013.

[3] Liver cancer fact sheet. Global Cancer Observatory, 2018. [Электронный ресурс] Режим доступа: http:/gco.iarc.fr/today/fact-sheets-cancers

[4] Clark T., Maximin S., Meier J., Pokharel S., Bhargava P. // Curr. Probl. Diagn. Radiol. 2015. V. 44. N 6. P. 479. doi 10.1067/j.cpradiol.2015.04.004

[5] Valery P.C., Laversanne M., Clark P.J., Petrick J.L., McGlynn K.A., Bray F. // Hepatology. 2018. V. 67. N 2. P. 600. doi 10.1002/hep.29498

[6] Mahvi D.A., Mahvi D.M. // Abeloff's Clinical Oncology. 2020. P. 846.

[7] Gharib H., Papini E., Paschke R., Duick D.S., Valcavi R., Hegedüs L., Vitti P. // Endocrine Practice. 2010. V. 16. Suppl. 1. P. 1. doi 10.4158/EP.16.3.468

[8] Pitman M.B. // Clin. Lab. Med. 1998. V. 18. N 3. P. 483. doi 10.1016/S0272-2712(18)30160-4

[9] Wee A. // Patholog. Res. Int. 2011. V. 2011. 587936. doi 10.4061/2011/587936

[10] Chhieng D.C. // World J. Surg. Oncol. 2004. V. 2. N 1. P. 5. doi 10.1186/1477-7819-2-5

[11] Choi S.H., Han K.H., Yoon J.H., Moon H.J., Son E.J., Youk J.H., Kim E., Kwak J.Y. // Clin. Endocrinol. (Oxf). 2011. V. 74. N 6. P. 776. doi 10.1111/j.1365-2265.2011.04011.x

[12] Gomez-Macias G.S., Garza-Guajardo R., Segura-Luna J., Barboza-Quintana O. // Cytojournal. 2009. V. 6. P. 9. doi 10.4103/1742-6413.52831

[13] Kalogeraki A., Papadakis G.Z., Tamiolakis D., KarvelaKalogeraki I., Karvelas-Kalogerakis M., Segredakis J., Moustou E. // Rom. J. Intern. Med. 2015. V. 53. N 3. P. 209. doi 10.1515/rjim-2015-0028

[14] Alfano R., Pu Y. // Lasers for Medical Applications. 2013. P. 325 .

[15] Кандурова К.Ю., Дрёмин В.В., Жеребиов Е.А., Альянов А.Л., Мамошин А.В., Потапова Е.В., Дунаев А.В., Мурадян В.Ф., Сидоров В.В., Крупаткин А.И. // Регионарное кровообращение и микроциркуляция. 2018. Т. 17. № 3. C. 71; Kandurova K.Y., Dremin V.V., Zherebtsov E.A., Alyanov A.L., Mamoshin A.V., Potapova E.V., Dunaev A.V., Muradyan V.F., Sidorov V.V., Krupatkin A.I. // Regional Blood Circulation and Microcirculation. 2018. V. 17. N 3. P. 71. doi 10.24884/1682-6655-2018-17-3-71-79

[16] Wang T.D., Van Dam J. // Clin. Gastroenterol. Hepatol. 2004. V. 2. N 9. P. 744. doi 10.1016/S1542-3565(04)00345-3

[17] Тучин В.В. Оптическая биомедицинская диагностика: в 2-х т. / Учебное издание. М.: ФИЗМАТЛИТ, 2007. Т. 1. $559 \mathrm{c}$.

[18] Kennedy G.T., Okusanya O.T., Keating J.J., Heitjan D.F., Deshpande C., Litzky L.A., Albelda S.M., Drebin J.A., Nie S., Low P.S., Singhal S. // Ann. Surg. 2015. V. 262. N 4. P. 602. doi 10.1097/SLA.0000000000001452

[19] Alchab L., Dupuis G., Balleyguier C., Mathieu M., FontaineAupart M., Farcy R. // J. Biophotonics. 2010. V. 3. N 5-6. P. 373. doi $10.1002 /$ jbio. 200900070
[20] Mayevsky A., Walden R., Pewzner E., Deutsch A., Heldenberg E., Lavee J., Tager S., Kachel E., Raanani E., Preisman S., Glauber V., Segal E. // J. Biomed. Opt. 2011. V. 16. N 6. P. 067004. doi 10.1117/1.3585674

[21] Evers D.J., Nachabe R., Hompes D., Van Coevorden F., Lucassen G.W., Hendriks B.H.W., van Velthuysen M.-L., Wesseling J., Ruers T.J.M. // Eur. J. Surg. Oncol. 2013. V. 39. N 1. P. 68. doi 10.1016/j.ejso.2012.08.005

[22] Spliethoff J.W., Prevoo W., Meier M.A.J., de Jong J., Klomp H.M., Evers D.J., Sterenborg H.J.C.M., Lucassen G.W., Hendriks B.H.W., Ruers T.J.M. // Clin. Cancer Res. 2016. V. 22. N 2. P. 357. doi 10.1158/1078-0432.CCR-15-0807

[23] Tanis E., Evers D.J., Spliethoff J.W., Pully V.V., Kuhlmann K., van Coevorden F., Hendriks B.H.W., Sanders J., Prevoo W., Ruers T.J.M. // Lasers Surg. Med. 2016. V. 48. N 9. P. 820. doi $10.1002 / 1 \mathrm{sm} .22581$

[24] Kandurova K., Potapova E., Shupletsov V., Kozlov I., Seryogina E., Dremin V., Zherebtsov E., Alekseyev A., Mamoshin A., Dunaev A. // Proc. SPIE. 2019. V. 11079. P. 110791C. doi $10.1117 / 12.2526747$

[25] Croce A.C., Bottiroli G. // Eur. J. Histochem. 2014. V. 58. N 4. P. 320. doi 10.4081/ejh.2014.2461

[26] Rafailov I.E., Dremin V.V., Litvinova K.S., Dunaev A.V., Sokolovski S.G., Rafailov E.U. // J. Biomed. Opt. 2016. V. 21. N 2. P. 025006. doi 10.1117/1.JBO.21.2.025006

[27] Harris K., Rohrbach D.J., Attwood K., Qiu J., Sunar U. // J. Thorac. Dis. 2017. V. 9. N 5. P. 1386. doi 10.21037 jtd.2017.03.113

[28] Braun F., Schalk R., Nachtmann M., Hien A., Frank R., Beuermann T., Methner F.-J., Kränzlin B., Rädle M., Gretz N. // Meas. Sci. Technol. V. 30. N 10. P. 104001. doi 10.1088/1361-6501/ab24a1

[29] Mathieu M.-C., Toullec A., Benoit C., Berry R., Validire P., Beaumel P., Vincent Y., Maroun P., Vielh P., Alchab L. // Eur. Radiol. 2018. V. 28. N 6. P. 2507. doi 10.1007/s00330-0175228-7

[30] Spliethoff J.W., Evers D.J., Jaspers J.E., Hendriks B.H.W., Rottenberg S., Ruers T.J.M. // Transl. Oncol. 2014. V. 7. N 2. doi 0.1016/j.tranon.2014.02.009

[31] Vo-Dinh T. Biomedical Photonics Handbook: Biomedical Diagnostics. CRC Press, 2014. 889 p.

[32] Mayevsky A., Rogatsky G.G. // Am. J. Physiol. Physiol. 2007. V. 292. N 2. P. C615. doi 10.1152/ajpcell.00249.2006

[33] Papayan G., Petrishchev N., Galagudza M. // Photodiagnosis Photodyn. Ther. 2014. V. 11. N 3. P. 400. doi 10.1016/j.pdpdt.2014.05.003

[34] Лукина М.М., Ширманова М.В., Сергеева Т.Ф., Загайнова E.B. // Современные технологии в медицине. 2016. Т. 8. N 4. C. 113; Lukina M.M., Shirmanova M.V., Sergeeva T.F., Zagaynova E.V. // Sovremennye Tehnologii v Medicine. 2016. V. 8. N 4. P. 113. doi 10.17691/stm2016.8.4.16

[35] Heikal A.A. // Biomark. Med. 2010. V. 4. N 2. P. 241. doi $10.2217 / \mathrm{bmm} .10 .1$

[36] Koenig K., Schneckenburger H. // J. Fluoresc. 1994. V. 4. P. 17-40. doi 10.1007/BF01876650

[37] Wang D., Chen Y., Liu J.T.C. // Biomed. Opt. Express. 2012. V. 3. N 12. P. 3153. doi 10.1364/BOE.3.003153

[38] Логинова Д.А., Сергеева Е.А., Крайнов А.Д., Агрба П.Д., Кириллин М.Ю. // Квант. электрон. 2016. Т. 46. № 6. C. 528; Loginova D.A., Sergeeva E.A., Krainov A.D., Agrba P.D., Kirillin M.Y. // Quantum Electron. 2016. V. 46. N 6. P. 528. doi 10.1070/QEL16133 
[39] Потапова Е.В., Дрёмин В.В., Жеребцов Е.А., Подмастерьев К.В., Дунаев А.В. // Фундаментальные и прикладные проблемы техники и технологии. 2018. Т. 331. № 5. С. 105. doi 10.1002/1873-3468.12964

[41] Foster K.A., Beaver C.J., Turner D.A. // Neuroscience. 2005. V. 132. N 3. P. 645. doi 10.1016/j.neuroscience.2005.01.040

[42] Zherebtsov E., Angelova P., Sokolovski S., Abramov A., Rafailov E. // Proc. SPIE. 2018. V. 10685. P. 106854E. doi $10.1117 / 12.2307552$

[43] Kim Y.J., Mizushima S., Tokuda H. // J. Biochem. 1991. V. 109. N 4. P. 616. doi 10.1093/oxfordjournals.jbchem.a123429

[44] Takahashi E., Endoh H., Ishikawa M., Kishi M., Doi K. // Oxygen Transport to Tissue XXIV. 2003. P. 565. doi 10.1007/978-1-4615-0075-9_54

[45] Drozdowicz-Tomsia K., Anwer A.G., Cahill M.A., Madlum K.N., Maki A.M., Baker M.S., Goldys E.M. // J. Biomed. Opt. 2014. V. 19. N 8. P. 86016. doi 10.1117/1.JBO.19.8.086016

[46] Bartolomé F., Abramov A.Y. // Methods Mol. Biol. 2015. V. 1264. P. 263. doi 10.1007/978-1-4939-2257-4_23

[47] Weissig V., Edeas M. Mitochondrial Medicine. V. 1. Humana Press, 2015. 480 p. doi 10.1007/978-1-4939-2257-4

[48] Mottin S., Laporte P., Cespuglio R. // Neurochem. 2003. V. 84. N 4. P. 633. doi 10.1046/j.1471-4159.2003.01508.x

[49] Dremin V., Potapova E., Zherebtsov E., Kozlov I., Seryogina E., Kandurova K., Alekseyev A., Piavchenko G., Kuznetsov S., Mamoshin A., Dunaev A. // Proc. SPIE. 2019. V. 108770. P. $108770 \mathrm{~K}$. doi 10.1117/12.2509255

[50] Lakowicz J.R. Principles of Fluorescence Spectroscopy. Springer Science \& Business Media, 2013. 698 p.

[51] The International Commission on Non-Ionizing Radiation Protection // Health Phys. 2004. V. 87. N 2. P. 171. doi 10.1097/00004032-200408000-00006

[52] Dremin V.V., Zherebtsov E.A., Sidorov V.V., Krupatkin A.I., Makovik I.N., Zherebtsova A.I., Zharkikh E.V., Potapova E.V., Dunaev A.V., Doronin A.A., Bykov A.V., Rafailov I.E., Litvinova K.S., Sokolovski S.G., Rafailov E.U. // J. Biomed. Opt. 2017. V. 22. N 8. P. 085003. doi 10.1117/1.JBO.22.8.085003.

[53] Плакунов В., Николаев Ю. Основы динамической биохимии. Логос, 2017. 216 с.

[54] Sivandzade F., Bhalerao A., Cucullo L. // Bio-protocol. 2019. V. 9. N 1. P. e3128. doi 10.21769/BioProtoc.3128

[55] Vargas G., Chan K.F., Thomsen S.L., Welch A.J. // Lasers Surg. Med. 2001. V. 29. N 3. P. 213. doi $10.1002 / 1 \mathrm{sm} .1110$

[56] Bui A.K., McClure R.A., Chang J., Stoianovici C., Hirshburg J., Yeh A.T., Choi B. // Lasers Surg. Med. 2009. V. 41. N 2. P. 142. doi $10.1002 / 1 \mathrm{sm} .20742$

[57] Capriotti K., Capriotti J.A. // J. Clin. Aesthet. Dermatol. 2012. V. 5. N 9. P. 24.

[58] Marren K. // Phys. Sportsmed. 2011. V. 39. N 3. P. 75. doi 10.3810/psm.2011.09.1923

[59] Pelzel H.R., Schlamp C.L., Waclawski M., Shaw M.K., Nickells R.W. // Invest. Ophthalmol. Vis. Sci. 2012. V. 53. N 3. P. 1428. doi 10.1167/iovs.11-8872

[60] Rojas J.C., Saavedra J.A., Gonzalez-Lima F. // Brain Res. 2008. V. 1215. P. 208. doi 10.1016/j.brainres.2008.04.001

[61] Hanslick J.L., Lau K., Noguchi K.K., Olney J.W., Zorumski C.F., Mennerick S., Farber N.B. // Neurobiol. Dis. 2009. V. 34. N 1. P. 1. doi 10.1016/j.nbd.2008.11.006

[62] Yu Z.W., Quinn P.J. // Biosci. Rep. 1994. V. 14. N 6. P. 259. doi 10.1007/BF01199051
[63] Galvao J., Davis B., Tilley M., Normando E., Duchen M.R., Cordeiro M.F. // FASEB J. 2014. V. 28. N 3. P. 1317. doi 10.1096/fj.13-235440

[64] Kirkpatrick N.D., Zou C., Brewer M.A., Brands W.R., Drezek R.A., Utzinger U. // Photochem. Photobiol. 2005. V. 81. N 1. P. 125.

[65] Danylovych H.V. // Ukr. Biochem. J. 2016. V. 88. N 1. P. 31. doi 10.15407/ubj88.01.031 